\title{
Characterization, analysis and recommendations for exploiting the opportunities of Cyber-Physical Systems
}

Authors:

- Martin Törngren; KTH Royal Institute of Technology, 10044 Stockholm, Sweden; martint@kth.se (corresponding author)

- Fredrik Asplund; KTH Royal Institute of Technology, 10044 Stockholm, Sweden; fasplund@kth.se

- Saddek Bensalem; Univ. Joseph Fourier Grenoble, Univ. Grenoble Alpes, VERIMAG, F-38000 Grenoble, France; saddek.bensalem@gmail.com

- John McDermid; Univ. of York, UK; Department of Computer Science, Deramore Lane, York, YO10 5GH; john.mcdermid@york.ac.uk

- Roberto Passerone; Dipartimento di Ingegneria e Scienza dell'Informazione - University of Trento, via Sommarive 9, 38123 Trento, Italy; roberto.passerone@unitn.it

- Holger Pfeifer; fortiss, Guerickestr. 25, 80805 Munich, Germany; pfeifer@fortiss.org

- Alberto Sangiovanni-Vincentelli; Univ. of California at Berkeley, EECS Department - 515 Cory Hall, Berkeley CA 94720; alberto@eecs.berkeley.edu

- Bernhard Schätz; Technical University of Munich, Boltzmannstr. 3, 85748 Garching, Germany / fortiss, Guerickestr. 25, 80805 Munich, Germany.

\begin{abstract}
:
Leveraging a comprehensive analysis of Cyber-Physical Systems (CPS) in Europe, this chapter presents overall findings focusing on (i) a characterization of CPS, (ii) opportunities and challenges in representative CPS application domains, and (iii) recommendations for action resulting from a cross domain analysis. The characterization enables a high-level description of a CPS, or classes of CPS, according to their technical emphasis, cross-cutting aspects, level of automation and life-cycle integration. We illustrate how these characteristics can be used to relate to design issues, systems and related terms.
\end{abstract}

The recommendations are to (1) Strengthen cross-disciplinary research collaboration, (2) Foster CPS education and training, (3) Stimulate public-private partnerships for CPS technology experimentation and to ensure dependable ICT infrastructure, (4) Promote interoperability of CPS technology, (5) Anticipate new business models and supporting open innovation, (6) Ensure trustworthiness including safety and security, and (7) Favor human-centered approaches to CPS.

Keywords: Cyber-Physical Systems, socio-technical systems, disruptive innovation, technological paradigm shift, automation, life-cycle integration, cross-domain integration, embedded systems, internet of things, systems of systems. 


\section{Introduction}

The findings described in this chapter draw on the CyPhERS project, an EU-funded support action that developed a strategy and agenda for CPS in Europe. This chapter summarizes the findings, focusing on (i) a characterization of CPS, (ii) opportunities and challenges in representative CPS application domains, and (iii) recommendations for action resulting from a cross domain analysis. The interested reader should consult CyPhERS project deliverables for details, starting with deliverables D6.1 and D5.2, Schätz et al. (2015) and Törngren et al. (2014), with further references therein.

As identified during the CyPhERS project, there are different interpretations of what constitutes a CPS, depending on what perspective is taken. The increasing connectivity, and penetration of electronics and software into all facets of our lives, is referred to differently by different research communities, such as Cyber-Physical Systems (CPS), Internet of Things (IOT), Ubiquitous Computing and the Fog and the Swarm; or labelled under application oriented terms such as Smart Cities or Industrie 4.0 (CPS in manufacturing). This led the CyPhERS project to devote a special effort to characterize CPS to complement existing definitions.

The core members of the CyPhERS project drew on their own expertise, and external expertise. Consequently, the project ran several workshops including consultations with a large number of experts, carried out state-of-the-art surveys, and undertook in depth analyses, including SWOT analyses, for five domains that were selected for investigation: Manufacturing, Health, Smart grid, Transportation and Smart cities.

CYPhERS had a broad remit and early on decided to go beyond a traditional technological focus. A major reason for the broader scope is due to the perceived disruptive nature of CPS and the potential ways in which CPS technology increasingly affects virtually all aspects of our society.

Based on the analyses of the five domains, CyPhERS conducted a cross-domain analysis to identify opportunities, challenges and strategies found to be common across the domains. This analysis led to the identification of high-level recommendations for action to grasp opportunities and deal with challenges.

In the remainder of the chapter we first provide the characterization of CPS, followed by an analysis of the five selected domains. We then proceed to describe the synthesized recommendations. Finally, we discuss the results and summarize conclusions. Overall, the chapter contains references for further reading (including the CyPhERS deliverables) and the discussion section provides references to related surveys.

\section{CPS characterization}

The term Cyber-Physical Systems (CPS), introduced in the US in 2006, was prompted by the increase of technical systems in which interactions between interconnected computing systems and the physical world are of primary importance. Early definitions illustrate how CPS are found both in the small and the large: "Such systems use computations and communication deeply embedded in and interacting with physical processes to add new capabilities to physical systems. These CPS range from minuscule (pace makers) to large-scale (the national power-grid)", CPS-summit (2008).

While such definitions make sense, they are generic; it is becoming increasingly difficult to identify systems which are not Cyber-Physical, given the increasing digitalization with penetration of electronics and software into virtually all facets of our lives. The concept of CPS ranges from massive to minimal systems. The concept is moreover inherently multi-disciplinary and multi-technological, and relevant across vastly different domains, with multiple socio-technical implications. The 
relevance of CPS thus remains difficult to evaluate for the uninitiated with respect to their impact and applicability to particular industrial sectors.

We therefore provide a high-level and minimalistic characterization of CPS using four perspectives that we deem of primary importance, namely, Technical emphasis, Cross-cutting aspects, Level of automation and Life-cycle integration. Our intention is to facilitate the description of particular CPS, or classes of CPS, of interest, and to provide a checklist to support planning and design of a CPS.

\section{CPS characteristics}

- Technical emphasis. CPS represent the integration of physical and embedded systems with communication and IT systems. With technical emphasis we refer to the technical part(s) of a CPS that are considered to be of particular importance, e.g. the embedded computing or the IT parts. When designing a CPS, there is a corresponding need to decide where emphasis should be placed, closely related to (i) how physical and embedded system parts are co-designed to enable optimizations and synergies, and (ii) how communication capabilities are used to off-load systems to enable cost reductions, optimized operations, etc. The most obvious impact of the associated design choices is on the scale and capabilities of a CPS, but there are also indirect business implications. As part of this characteristic we also encompass the considered scale of a CPS to further clarify the focus.

- Cross-cutting aspects. With cross-cutting aspects we refer to system properties (such as safety and security), jurisdiction (i.e. applicable standards and legislation) and governance (i.e. where responsibility lies for the safe, efficient, secure operation of the system). These aspects thus refer to the constraints for operation and organizational responsibilities in meeting those constraints. The advances in connectivity make it possible to create new applications that span several traditional application domains. This opens up new business opportunities, but also requires that technical and non-technical "gaps and barriers" across the domains are dealt with. While connectivity may be desirable it also necessitates explicit consideration of properties such as security. Adaptability across different environmental contexts and use cases is often driven by business considerations (e.g. reduced maintenance costs and increased availability), and may eventually require dynamic reconfigurability.

- Level of automation. Designing a CPS involves careful investigation to ascertain a suitable level of automation. With level of automation we refer to what activities are automated and to what degree, Parasuraman, Sheridan and Wickens (2000). The increasing interest in autonomous vehicles has driven the development of classifications of levels of automation, e.g. the standard for automated driving established by the Society for Automotive Engineers, (2014). CPS are typically designed to act more or less independently of humans, even if they may be triggered by human inputs, or interact closely with humans, including shared control. Shared control also has its challenges: it is crucial to clarify who is in control at any point in time to make sure that unintended control does not take place, implying that human-machine interface design is often crucial. The level of automation closely corresponds to notions such as adaptability and to some extent corresponds to the "smartness" of a CPS.

- Life-cycle integration. Life-cycle integration is driven by quality, cost and business concerns. With life-cycle integration we refer to a spectrum, from a CPS with no integration in the management of the product, services and data over the life-cycle, to full integration of development and operations, including capabilities to upgrade and collect data from an operational system. The 
resulting trade-off concerns the benefits vs. the costs of investments to ensure integration between the various IT systems (e.g. the engineering environments) and with the product in operation.

In describing CPS, the characteristics help to clarify what type of system that is considered, also with regard to different stakeholders and viewpoints. The characteristics in addition serve to set the ambition in CPS design, e.g. regarding the desired level of automation. The characteristics are applicable for both small and large CPS.

Many terms have been coined to mirror the opportunities enabled by connectivity and computing. These terms largely provide similar messages but from different perspectives. The CyPhERS project contrasted CPS with such other terms. We here provide two examples of this based on the characteristics and refer interested readers to Törngren et al. (2014) for further examples:

IoT emphasizes sensing of the physical world and uniquely identifiable things with (internet) connectivity, that communicate data with limited or no human interaction. Communication is often considered the key aspect - thus providing a specific technical emphasis. CPS differs through a systems perspective, not necessarily requiring internet connectivity.

Systems of Systems (SoS) usually address the construction of evolving large-scale systems and the coordination among those systems, specifically focusing on integration and optimization to satisfy a wide range of objectives. The concept of SoS is independent of the type of system (e.g. organizational or socio-technical). Many SoS will indeed incorporate CPS, and may also themselves be considered as CPS (as long as one can reconcile the terms system and SoS). The cross-cutting aspects of a CPS will largely characterize whether the actual CPS is a SoS or not.

\section{Further CPS characterization through market analysis}

CPS have the potential to be disruptive - to substantially change the nature of markets. This can be through creation of new markets or through substantial changes of ecosystems. CyPhERS developed a market analysis method, to try to identify the potential for CPS to shape markets - whether disruptive or transformative, McDermid et al. (2014). The method, which complements the previously described characteristics, includes an analysis of opportunities and constraints at each of four "layers": social, process, information and technology, see Figure 1; this model is known as the SPIT model, Sillitto (2010). CPS are anchored in the technology and information layers. For example, innovations at the information layer may allow horizontal integration by drawing on the sensing capabilities of CPS. Such integration may in turn enable new business models at the process level. Innovation and constraints can arise at any level, but the constraints at the social level are important, as what is possible might not be socially acceptable.

An initial analysis of the five domains studied in the CyPhERS project came to the conclusion that disruption is unlikely in the domain of smart grids, but much greater changes are possible in the other domains, McDermid et al. (2014). While these findings need further in-depth analysis to be validated, we found the SPIT model useful for reasoning about the role of CPS in socio-technical systems including assessing potential business impact.

\section{Analysis of representative CPS domains}


The analysis of opportunities and challenges in the CPS domains was carried out as a comprehensive SWOT analysis, Törngren et al. (2014). This section summarizes opportunities and challenges identified during the analysis, based on the mentioned CPS characteristics.

\section{CPS in manufacturing}

The industrial domains and processes of Manufacturing, representing a major socio-economic force, are strongly characterized by the use of CPS technologies. Manufacturing encompasses CPS with different types of technical emphases, from 3D scanners/printers to cloud manufacturing. The increased emphasis on IT integration and openness means that security, as a cross-cutting property, is becoming increasingly important. Manufacturing has been a forerunner in automation with solutions transferred to other domains, e.g. from industrial robotics to autonomous vehicles. Mass customization is currently driving the development of more flexible and efficient production systems, see e.g. Wang et al. (2015). Advanced industrial companies have already introduced life-cycle integration - tracing real operational data back to development and manufacturing. This trend is likely to continue. Manufacturing as a domain, has also to some extent been integrated with other domains, primarily with transportation for logistics, providing cross-domain solutions. Opportunities arise from new business models involving open innovation, paving the way for flexible, customizable distributed manufacturing schemes.

At the societal level, it is essential that sustainability be taken seriously. We note for example that about $14 \%$ of the total 2,652 million tons of waste that were generated in EU-27 countries in 2008 were due to manufacturing, Eurostat (2011). CPS technology provides solutions that assist in dealing with sustainability, such as modular architectures to facilitate reuse and recycling.

Complex CPS will feature prominently in future manufacturing systems. The management of such systems, dealing with security and safety risks, and providing efficient interoperability, poses barriers to their successful industrialization. Lack of the new competences required may prevent successful industrial evolution; in particular the provision of additional skill sets encompassing internet, security and software are seen as a key enablers.

\section{CPS in healthcare}

CPS in healthcare have varied applications and technical emphases, from medical devices to improve the efficacy of medical treatment and surgery, to remote services based on collected data. The paradigm shift in level of automation from what used to be essentially passive devices, controlled by human operators, to IT-enabled devices is significant. Emerging health-care devices and equipment actively control critical physiological processes and functions. The embedded computing, sensing, modeling, communications, and deep integration with physical elements and processes allow these new CPS to achieve levels of functionality, adaptability, and effectiveness not possible with simpler passive systems, NITRD (2009), HMGov (2013).

A widespread adoption of CPS will be able to provide data of unprecedented size and accuracy regarding the effectiveness of treatment, giving doctors invaluable information for fine-tuning processes and procedures to achieve better life-cycle integration for both products and patients. Similarly, a better understanding of the side conditions and real-time information is essential to personalize treatments and achieve better outcomes. In particular, CPS have the potential to reach the body using minimally or non-invasive techniques, which lower costs and enhance mobility, independence and quality of life. The continuous monitoring of a chronic condition has also the potential of substantially shifting care delivery from inpatient to outpatient services and to the home. 
The diversity and interconnection, coupled with the sensitive nature of dealing with life-related conditions, makes the design of CPS in healthcare challenging and leads to severe cross-cutting issues. While data collection is essential to improve healthcare services, its security and privacy must be guaranteed, and devices must be immune to attacks, as they may provide access to the body. At the same time, these systems are complex and require new technologies, which have had only limited testing, leading to malfunctions, due primarily to design failures, but also due to materials and components, Admet (2014). This highlights the challenges faced by the design process, which must be supported by new methodologies and tools, Davare et al. (2013). In addition, healthcare is regulated to guarantee, through certification, the introduction of safe and effective treatments. However, increasingly strict regulations and longer clinical trials could have significant impact on cost and investments. In Europe in particular, regulations are not homogeneous among the member states, leading to uncertainty and risk for the industry.

\section{CPS and smart grids}

Infrastructures providing reliable access to energy form a foundation for our industrialized societies. Traditionally, an electric grid is implemented by a small number of high-volume facilities on the production side and large number of generally low-to-medium volume installations on the consumer side, with a varying demand of a factor of four between lows and peaks during the day. The increasingly used renewable energy resources are generally produced by a larger number of facilities with mostly volatile volumes not in synch with the requested consumption, rendering the traditional asymmetric and centralized management scheme of the electric grid increasingly inadequate, see e.g. Hashmi (2011). Here, the new technical emphasis of the CPS of a smart grid offers a solution by enabling the decentralized and cooperative coordination of technical and organizational processes, from the control of a photovoltaic installation to the billing and trading of energy. Such decentralized solutions enable microgrids (local grids) that can operate with or without a connection to the main grid.

Similarly, automation that monitors and predicts consumption as well as production of renewables, via smart meters on a fine-grained level, facilitates a reliable short-term balancing of demand and supply. The use of intelligent devices and managed installations including low-volume energy buffers (e.g. batteries), support a shift from a supply- to a demand-side management of the grid. The highlyautomated control of a CPS allows these processes to be scaled to the required number of participants.

A life-cycle integration that allows this control to be updated seamlessly across an entire smart grid promises even further gains. Locally inefficient control can be identified and replaced seamlessly, and additional data or distributed sensing capabilities added in a modular fashion. Furthermore, if data is shared between the manufacturers and users of CPS of smart grids, further opportunities for customization can be identified.

However, this will not happen if the necessary technological (including interoperable, safe and secure infrastructure) and regulatory prerequisites (including suitable tariffs, market models and transnational grid operation schemes) cannot be established. The ability to understand the crosscutting implications of producing, trading in and monitoring energy must be solid enough, so that uncertainty and risk does not prevent distributed investments into smart grid infrastructure.

\section{CPS in transportation}

Transportation encompasses CPS, all the way from smart components such as a smart tyre to intelligent transportation systems. As transportation systems are growing to meet the future demands of society, they are evolving towards increasingly complex SoS, as exemplified by the new 
European Rail Traffic Management System (ERTMS). ERTMS encompasses CPS with a new technical emphasis through a dedicated GSM communication system that connects trains, infrastructure systems and system management.

Transportation systems have a direct relation to cross-domain integration. Transportation services require the coordination of processes across sectors like logistics, automotive and rail. The coordination is influenced both by the corresponding vehicles and infrastructural components (e.g. roads and communications), with differences including speed, capacity, cost, and governance.

With logistics being an integral part of industrial and societal processes, the shift from mobility as the provision of vehicles to mobility as a service is accelerating. Modern mobility solutions will increasingly focus on highly automated forms of transport, addressing the need for individual transport without the need for an individually owned vehicle. The vision of automated guided and connected vehicles is not only necessary to cope with the increased demand for mobility, but also address the additional societal goals of increased safety, efficiency, security, convenience, and the economy, Sussman (2005). An important factor is the requirement to adapt transport services to an increasingly ageing population: quality, reliability, security, accessibility for persons with reduced mobility, and safety are essential to meet this requirement with public transport.

While most CPS domains share similarities in the challenges they face, there are several unique problems linked to transportation CPS. The customization and variability of system architecture weaves a level of complexity rarely observed. The ultra-competitive global landscape mandates everevolving requirements for enhanced capabilities, resulting in the need to rapidly adapt systems. The changing landscape leads to an increased importance of cross-cutting aspects such as safety and security; and the evolving need for life-cycle integration to allow for pattern analysis of accident statistics, continuous updates to vehicles to remove defects or avoid unsuitable driver behavior, etc. In addition to the competition in the pursuit of vehicle consumers, the global competition at the component level is equally fierce, resulting in a diverse and constantly evolving set of component suppliers that must provide products to be integrated into the whole.

\section{CPS and smart cities}

Smart Cities involve the integration of many domains of CPS research and technology. In addition, they touch other domains such as architecture and legal, economics and social sciences - they truly place emphasis on the cross-domain aspects of CPS. A background paper by the UK Department for Innovation and Skills provides a good summary of the challenges and opportunities that cities and business are facing when inserting digital technology into cities, UKBus (2013).

Opportunities for CPS in smart cities abound due to the present situation. In Europe many of the cities date back to the Roman times. This results in an often chaotic layout of the downtown areas with narrow and winding streets. Traffic is at times unmanageable. In the US, the situation is more critical in other respects, such as the large number of cars in the urban highway systems. Without the determined use of advanced technology with a new technical emphasis the situation will be unmanageable. The deployment of a capillary network of sensors and of pervasive communication typical of CPS allows monitoring and controlling security, safety and efficiency in smart cities as well as the development of new services to make cities more livable. A high degree of automation of future city services will provide an unprecedented ability to avoid wasting resources when directing city transports; losses linked to water pollution, fire or the release of hazardous materials; and disturbances due to failing infrastructure.

The most serious challenges are related to decision makers, often unaware of technology. Whenever confronted with a plan to add a CPS driven infrastructure or service, they may fall prey of unwise 
designs. Indeed, there have been examples of technology insertions that ended up in totally wasted money and effort, Greenfield (2013). An increased level of life-cycle integration, where data is harvested and certain smart city technology proven to be linked to benefits, will be required to allow decision makers to act (almost) regardless of their level of technological expertise.

\section{Recommendations based on a cross-domain analysis}

The SWOT analysis for CPS domains was followed by a cross-domain analysis. This analysis was used to identify patterns (challenges, opportunities, strategies) common across the domains, Törngren et al. (2014). Due to space limitations, we focus on the common recommendations across domains.

Strengthen cross-disciplinary research collaboration: The need to strengthen key research in CPS is common across most CPS domains since current approaches to design and verification are already stretching the limits for cost-efficient system development; there is an urgent need to update the engineering methodologies for CPS. There is a need to develop funding schemes that to a greater extent stimulate the creation of truly multidisciplinary consortia, bridging the gaps between traditional disciplines, e.g. embedded systems vs. internet and big data, and between application domains. Corresponding strategies include support of broader networks of excellence, and to stimulate learning networks among industrial domains.

Foster enabling education and training: Excellence in education and a skilled work force is of paramount importance for exploiting CPS opportunities. The problem is the growing amount of knowledge and skills required for product and service engineering. In order to create engineers capable of building CPS, education must break the disciplinary silos, and provide cross-disciplinary technology and project experiences. Incentives are needed to stimulate academia and industry collaboration in education. To ensure the necessary re-qualification, an academic-industrial alliance should be formed to support engineers in life-long learning.

Stimulate public-private partnerships for CPS technology experimentation to deal with societal challenges and to ensure a dependable information and communication infrastructure: The adoption of key CPS technologies will depend on their maturity, requiring their application in realworld and large-scale installations through maturation initiatives. The level of complexity introduced by CPS further mandates experimental and incremental approaches to system realization. Publicprivate partnerships are needed to ensure the availability and affordability of dependable and trustworthy information and communication infrastructure.

Promote interoperability of CPS technology through reference platforms and standards: CPS depend critically on integration. Public incentives are needed to facilitate interoperability across the engineering life-cycle, and within and across domains and disciplines. Interoperability goes beyond technology and requires consideration of, for example, concrete business drivers and regulations to make sure that "standards" are developed at the right level. This amounts to providing reference platforms to support the integration of services as well as homogenizing interoperability standards. Whilst this activity must be led by industry, regulators and other public bodies should encourage and support these initiatives.

Prepare for disruption by anticipating new business models and supporting open innovation: New value-added end-user services will become important 'products' in the context of CPS and will give rise to new business models and ecosystems (for example by selling transport services rather than vehicles). To stimulate such ecosystems, forums should be provided facilitating contacts and collaboration among innovators trying to enter the service ecosystem of a CPS and existing providers of services. Research and innovation should also stimulate the development of, and research into, new business models. The orchestration of basic services will often rely on established and costintensive infrastructures where innovation opportunities will depend on easy access to those services. Funding programs must therefore promote open standards, the provision of open-source or 
open/free license results, and promote interoperability. Opportunities for big-data analytics require well-defined open data access as well. In order to reduce entry barriers for innovative enterprises clear liability regulation frameworks must be provided and corresponding supporting technologies must be put into place that help to identify acceptance and delegation of responsibilities for services provided.

Ensure trustworthiness including safety and security: The pervasiveness of CPS implies that their malfunction or misuse can have dramatic negative effects on society and the economy. Safety and security are exposed as intertwined and truly cross-cutting issues. They require revised standards and regulations and the development of new engineering methodologies to ensure that the implemented systems meet agreed-upon trust levels. Security requires special consideration as previously closed systems become exposed in new ways. Joint public and private investments are needed to assess and improve the security of both public and private information and communication technology to protect these critical infrastructures from cyber-attacks. There is a strong human element here - it is humans who will determine whether or not a CPS-based system is to be trusted.

Ensure that humans are at the center of approaches to CPS: Because societies will rely on CPS it is of paramount importance not only that they are effectively engineered, but also well understood and appropriately used. Overall, ensuring human-centered approaches to CPS requires that related efforts, from training to research and experimentation, need to include and consider a broader set of stakeholders than just engineers and system developers. Essentially, a very broad set of stakeholders, including policy makers and the general public, will need a basic understanding about CPS implications in terms of both opportunities and risks. A further important concern is to address the missing cross-fertilization between engineering sciences and humanities. We cannot afford this gap to continue for societal level CPS systems. This becomes even more important with the increasing level of automation provided by CPS functionalities. Finally, there is an urgent need to pay explicit attention to sustainability and privacy with consideration of related trade-offs, for example referring to data sharing vs. privacy, and openness vs. security threats. Economic, social and environmental sustainability considerations need to be explicitly promoted in CPS initiatives to deal with the embedding of digitization everywhere. The pervasiveness of CPS in social processes, demands built-in mechanisms to protect the privacy of its users, but also raised awareness of those users in interacting with CPS. To avoid misuse of sensitive data acquired by CPS, the establishment of regulations clarifying data ownership including granting and revoking access, as well as corresponding technical implementations are necessary.

\section{Discussion and related work}

The SWOT analysis formed one important background for the recommendations. Several interactions with a wide range of stakeholders and other research initiatives took place to validate the findings. We believe the end results to be valid in that they represent strategic areas for Europe (although not necessarily valid for each European region). We also believe that many of the recommendations would be valid also for regions beyond Europe; however, making such claims for specific regions requires further validation.

Our recommendations were common across all domains albeit with different emphasis in some. The need to achieve better understanding of cross-cutting aspects and domain integration deserves special attention; engaging relevant stakeholders in debate and as part of pilot trials will be very important.

While regulations were not highlighted as an explicit recommendation in this chapter, we would like to emphasize the importance to evolve and harmonize regulations related to CPS, in order not to impose over-constraining barriers. 
As CPS draw upon many different fields of technology, unsurprisingly there is a partial overlap of the identified recommendations with strategic agendas from these domains, most specifically those targeting complex embedded and networked systems, for example ITEA-ARTEMIS (2013), and the ARTEMIS strategic research agenda, ARTEMIS-SRA (2013). Unlike those, CyPhERS took a broader approach including societal, market and education aspects. There is also a partial overlap concerning the recommendations with other national agendas, most specifically the recommendation from the US CPS Summit report, CPS-summit (2008), and the German agendaCPS, Acatech (2012). Despite slightly different focus (regions and domains), it is notable that the findings overall point in similar directions.

The interest in CPS is seen from a large number of publications including text books such as Lee and Seshia (2015) and Alur (2015). For the interested reader, the comprehensive survey of CPS technologies and applications by Khaitan and McCalley (2015) provides further useful references. The paper by Fisher et al. (2013) reviews scientific and engineering challenges of CPS.

As a complement to our high-level characterization of CPS, more detailed frameworks include the ones by Baras J and Austin M (2013) and the CPS-PWG (2015).

\section{Conclusions}

CPS are characterized by integration, across technologies, industrial domains and the life-cycle, and by "smartness". CPS can be described using a corresponding set of characteristics: Technical emphasis, Cross-cutting aspects, Level of Automation and Life-cycle integration.

CPS, intended as the integration of cyber and physical parts, is not a new concept, but is now increasingly manifesting itself in terms of larger scale integrated systems that provide unprecedented opportunities for innovation.

Exploiting the opportunities made possible by CPS requires overcoming a number of challenges including developing scientific and engineering methodologies that cater for the complexity of CPS, providing dedicated education and training to relevant stakeholders, preparing for evolving business models, ensuring trustworthiness, as well as dealing with societal and legislative challenges.

The recommendations we described in this chapter are geared to address these challenges. Electronics is already being embedded "everywhere" in our societies. CPS will pave the way for even more digitalization. CPS further creates important business opportunities for largely automated systems. The implication is that economic, social and environmental sustainability must be considered now in order to ensure that planning, adoption and deployments sufficiently consider these aspects, in turn ensuring that humans remain at the center stage of a CPS-based society.

\section{Acknowledgements}

This work was support by the European Commission through the CyPhERS FP7 support action (contract no. 611430) and the CPSELabs Innovation action (contract no. 644400). We acknowledge contributions from Maria-Victoria Cengarle and Thomas Runkler, who were part of the CyPhERS projects, together with inputs and feedback from numerous experts who contributed to the CyPhERS efforts.

\section{References}

Acatech, 2012, agendaCPS - Integrierte Forschungsagenda Cyber-Physical Systems. E. Geisberger, M. Broy (eds.), Springer-Verlag, 2012. English translation available as: Living in a networked world. Integrated research agenda Cyber-Physical Systems (agendaCPS). E. Geisberger, M. Broy (eds.), acatech, 2015. Available online 
http://www.acatech.de/de/publikationen/publikationssuche/detail/artikel/living-in-a-networkedworld-integrated-research-agenda-cyber-physical-systems-agendacps.html (accessed Sept. 2015).

Alur R, Principles of Cyber-Physical Systems, MIT Press, 2015.

ARTEMIS-SRA, 2013, Embedded/Cyber-Physical Systems ARTEMIS Major Challenges: 2014-2020 2013 Draft Addendum to the ARTEMIS-SRA. URL: www.artemisia.eu/publication/download/publication/910/file/ARTEMISIA SRA Addendum.pdf (accessed Sept. 2015).

Baras J and Austin M (2013), Development of a framework for CPS open standards and platforms, Institute for Systems Research, Univ. of Maryland, technical report 2014-02.

Cengarle MV, Bensalem S, McDermid J, Passerone R, Sangiovanni-Vincentelli A and Törngren M, 2013, Characteristics, capabilities, potential applications of Cyber-Physical Systems: a preliminary analysis, Deliverable D2.1 of the CyPhERS FP7 project, Nov. 2013. http://www.cyphers.eu/sites/default/files/D2.1.pdf

CPS-PWG (2015), Framework for Cyber-Physical Systems, Draft, Release 0.8, September 2015, Cyber Physical Systems Public Working Group, an open public forum established by the National Institute of Standards and Technology (NIST), https://pages.nist.gov/cpspwg/ (accessed Sept. 20th 2015)

CPS-summit, 2008, Holistic Approaches to Cyber-Physical Integration, CPSWeek Report - CyberPhysical Systems Summit, 2008. URL: iccps2012.cse.wustl.edu/_doc/CPS_Summit_Report.pdf (accessed Sept. 2015).

Admet 2014, Medical device sector review, ADMET Inc. Norwood, MA. May 2014. URL: http://www.onlinetmd.com/FileUploads/file/ADMET_Medical_Device_Sector_Review.pdf, (accessed Sept. 2015).

Davare A, Densmore D, Guo L, Passerone R, Sangiovanni-Vincentelli A, Simalatsar A and Zhu Q, 2013, metroll: A Design Environment for Cyber-Physical Systems, ACM Transactions on Embedded Computing Systems, 12(1s):49:1-49:31, March 2013.

Eurostat, 2011, Eurostat pocketbooks- energy, transport and environment indicators, 2010 edition. Technical report, European Union, 2011.

Fisher A, Jacobson C, Lee EA, Murray R, Sangiovanni-Vincentelli A, Scholte E, Industrial Cyber-Physical Systems - iCyPhy, Proc. Complex Systems Design \& Management (CSD\&M), Springer, 21-37, 4, Dec. 2013, Paris, France.

Greenfield A, 2013, Against the Smart City, New York: DO Projects.

Hashmi M, 2011, Survey of Smart Grids Concepts Worldwide, Technical Report, VTT Working Papers 166, VTT, January 2011. URL: http://www.vtt.fi/inf/pdf/workingpapers/2011/W166.pdf (accessed Sept. 2015).

HMGov, 2013, Strengths and opportunity 2013, Technical report, HM Government, 2013. URL: https://www.gov.uk/government/uploads/system/uploads/attachment_data/file/298819/bis-14p90-strength- opportunity-2013.pdf (accessed Sept. 2015).

ITEA-ARTEMIS, 2013.ITEA ARTEMIS-IA High-Level Vision: Opportunities for Europe. 
Khaitan SK and McCalley JD, 2015, Design Techniques and Applications of Cyberphysical Systems: A Survey, IEEE Systems Journal, Vol. 9, No. 2, June 2015.

Lee EA and Seshia SA, 2015, Introduction to Embedded Systems, A Cyber-Physical Systems Approach, $2^{\text {nd }}$ edition, http://LeeSeshia.org, ISBN 978-1-312-42740-2.

McDermid J, Cengarle MV, Törngren M, Runkler T, 2014, Market and innovation potential of CPS, Deliverable of the CyPhERS FP7 project, August 2014 (available on-line: URL: http://www.cyphers.eu/sites/default/files/D3.2.pdf).

NITRD, 2009, High Confidence Software and Systems Coordinating Group, High-confidence medical devices: Cyber-physical systems for 21st century health care. Technical report, Networking and Information Technology Research and Development Program, February 2009.

Parasuraman R, Sheridan TB and Wickens CD, 2000, A model for types and levels of human interaction with automation, Systems, Man and Cybernetics, Part A: Systems and Humans, IEEE Transactions, 30(3): 286-297.

Sillitto H, 2010, Design Principles for Ultra-Large Scale (ULS) Systems, INCOSE International Symposium, Proceedings, pages 63-82.

Society for Automotive Engineers, (2014), Taxonomy and Definitions for Terms Related to On-Road Motor Vehicle Automated Driving Systems, Surface Vehicle Information Report, J 3016, no. 01.

Schätz B, Törngren M, Bensalem S, Cengarle MV, Pfeiffer H, McDermid J, Passerone R, SangiovanniVincentelli A, 2015, Research Agenda and Recommendations for Action, Deliverable of the CyPhERS FP7 project, March 2015, (available on-line: http://cyphers.eu/sites/default/files/d6.1+2-report.pdf)

Sussman, J, 2005, Perspectives on Intelligent Transportation Systems, Springer.

Törngren M, Bensalem S, Cengarle MV, McDermid J, Passerone R and Sangiovanni-Vincentelli A, 2014, CPS: Significance, Challenges and Opportunities, Deliverable D5.2 of the CyPhERS FP7 project, Dec. 2014 (available on-line: . http://www.cyphers.eu/sites/default/files/D5.2.pdf)

UKBus, 2013. Smart cities: background paper, Department for Business Innovation and Skills of the UK, available on-line at: https://www.gov.uk/government/uploads/system/uploads/attachment data/file/246019/bis-131209-smart-cities-background-paper-digital.pdf (accessed Sept. 2015).

Wang L, Törngren M and Onori M, 2015, Current Status and Advancement of Cyber-Physical Systems in Manufacturing, Journal of Manufacturing Systems, Vol.37, Part 2, pp.517-527. 